\title{
Exploration on application of optical communication technique to 4G technological development
}

\author{
Chunmao Xie
}

\author{
College of Physics and Electronic Information, China West Normal University, Nanchong, 637000, \\ China
}

Keywords: 4G technical development; Optical communication technique; Exploration on application

\begin{abstract}
GLTE network was launched by Verizon Company and they promised that they would improve $4 \mathrm{G}$ network speed to a higher level and try hard to make the speed be 10 times higher than that of $3 \mathrm{G}$ network. As a brand-new communication form, optical communication technique has appeared in our life in recent years. Since the technique develops relatively late, it does not develop rapidly as people imagine but experiences many setbacks. However, the technique still plays a huge role in the process of $4 \mathrm{G}$ technological development and is an important guarantee on which $4 \mathrm{G}$ network can obtain effective development. In order to promote effective development of $4 \mathrm{G}$ network technology, the article elaborates related content in the following passages.
\end{abstract}

\section{Introduction}

In 2013, deployment of $2 \mathrm{G}$ network showed a declining trend in some developing countries of the whole world. However, they did not give up input into network technology but upgraded current network level continuously to build the network that could replace $2 \mathrm{G}$ network and operate faster. In doing so, some new requirements are proposed for optical communication technique. In detail, it is required that network switching should have stronger functions and higher speed, network construction of cost efficiency should be promoted and current network should be more intelligent. Meanwhile, some new techniques and products are launched continuously and it is expected that they can enter some new fields as people's cognition about optical property deepens constantly. For this, the article centers on specific development background of the technique and elaborates its detailed situations in $4 \mathrm{G}$ development.

\section{Review and prospect on development of optical communication}

Optical communication is an important type in communication technology. This communication mode mainly uses optical wave as a medium. The communication technology that treats optical communication technique as a premise is a main feature of optical communication technique. Optical communication technique plays an important role in communication network where optical communication is an important component. Development prospect of communication network is decided by its development degree to some extent. Thus, importance of the technique is obvious. Next, the article will utilize the following passages to introduce and analyze the three techniques ASON, ROADM and OADM qualitatively and specifically.

The technique ASON has high flexibility and high expansibility, which is optical network that can provide service directly according to demands in optical layer and composed of management control and optical transport network and optical signal control network. Perfection of intelligent optical layer network nodes and control plane is a key to realization of ASON, for instance, realization of wave length router, OXC and OADM. Meanwhile, a brand-new concept CP is proposed in the technique. CP mainly relates to interface, protocol and signaling and is primarily responsible for connection, maintenance and management of network resources.

We may also use English about optical add-drop multiplexer to describe OADM. Its main function is to realize add-drop and multiplex of branch signals. Besides, main techniques of OADM contain OTDM, WDM and O-CDMA. In all optical network, optical add-drop multiplexer is one of 
its critical devices and exists in all optical network techniques with great importance.

Reconstructed optical add-drop multiplexer is a main feature of add-drop multiplexer, which utilizes re-configured remote equipment and information and then can use dynamic methods to control wave length between upper and lower business effectively. Besides, it may manage wave length of business effectively. It can be called node or element of serious network. Although ROADM may provide a series of advantages and superiorities for network service, for instance, the superiority that management may be more automatic, the second-line position is still a main form that ROADM faces with according to analysis of current situations. Price of ROADM is a main reason for this phenomenon. Thus, development of ROADM is limited and restricted by its high price to some extent.

\section{Analyzing related content of $4 \mathrm{G}$ communication technique}

1. Orthogonal frequency division multiplexing (OFDM)

OFDM is main technical support for $4 \mathrm{G}$ communication technique. Its main though is that information channels are changed into the form of several sub-channels. Low-speed streaming data is transported concurrently and signals of high-speed data can be converted. In this way, all sub-signals can be unblocked. Proportion of channel bandwidth of each sub-channel to signal bandwidth is not large, which can prevent mutual interference among channels effective and eliminate inter-symbol interference well. OFDM has many advantages, such as anti-fading ability, good adaptation to transportation of high-speed data, ability to resist code interference and capability of high spectral efficiency etc.

2. Intelligentized jug line function

Without multi wave velocities of wave velocity switching or the intelligent jug line that adapts to array jug lines, intelligent jug lines are considered to be key technique in future mobile communication since they have powerful functions, such as adjusting data wave velocity, restraining signal interference and automatic tracking and can restrain mutual interference in airspace efficiently. Besides, it can improve capacity of transportation and change signal quality effectively. Its basic principle is that it uses base band digital processing unit, integrates signals accepted on jug line links according to some compute modes and realizes ascending wave constraint.

3. Core network based on IP

The said 4G system is generated based on IP network, which can realize seamless interconnection of different kinds of network effectively, be independent of some specific wireless access schemes and switch many types of air network into core network and separate transportation of core network business from control. If IP is used, protocol, link layer and access method will be independent separately.

\section{Analyzing specific application of optical communication technique to $4 \mathrm{G}$ network development}

1. Being applied to optical transport network and OTN

Although the largest wireless transmission bandwidth of $4 \mathrm{G}$ and its frequency point will cause some restrictive effect, there is some bandwidth pressure at the initial stage of $4 \mathrm{G}$ network development since some restriction factors exist in data rate of users. The statement that larger particles need be applied to bandwidth has been proposed. Therefore, we should have a specific solution about expandable, reliable and effective network transmission. Since OTN technique is generated gradually as demands are put forward and after related work has been studied, some driving force is provided for $4 \mathrm{G}$ network development. A main advantage and feature of OYN technique system is that it is integrated, systematic and comprehensive system which integrates many high and new technologies and can realize nearly all existing demand functions at present, construct systematic and complete electrical property layer and optics as well as contribute to realization of monitoring about the whole network. Besides, it can realize existing mechanism in 
two-layer network well. According to development history and situations of the whole technique, we may clearly find that it is a result of effective integration of WDH technique's and SDH technique's functions and advantages.

2. Being applied to intelligent optical network layer

Mobile service network has significant differences in user distribution and specific network transmission in the next generation of $4 \mathrm{G}$ mobile network. In accordance with detailed comparison with 3G network, it may be found that base flow with different features exist in $4 \mathrm{G}$ network. Accordingly, features like strong burstiness and dynamic nature exist, and there are higher requirements for network transmission. On the premise that there is cross processing is not blocked in the process of some intelligent optical network of connecting circuits, dynamic route control is integrated with this organically so that development from a static state to a dynamic state in the process of network transmission and powerful gurantee can be provided for $4 \mathrm{G}$ mobile network effectively. The data transmission network rising based on $4 \mathrm{G}$ business improves dynamic scope largely, so flexible demands will be enhanced gradually. Therefore, it can be said that intelligent optical network will be an important gurantee and premise for its effective development in the development process of $4 \mathrm{G}$ mobile network.

3. Being applied to carrier-class Ethernet

At present, Ethernet technique enters a brand-new development stage and has been widely applied to reality. Especially, its application frequency in telecommunication network is improved continuously. Since it is essential to adapt to various demands of telecommunication network under the background that information technology and mobile network data are developed and improved constantly, Ethernet technique appears in our life and work under such a background. ASON and SDH have walked towards a mature direction up to now. Thus, we may consider combining it with ASON technique or applying it to MSTP technique perfectly on this premise. Under the situation that maximum horizontal force can realize 128 bits per second in network nodes, we may study and develop ASON control plane and improve the schedule mentioned in OTN continuously. Currently, efficient photoelectric cross layer also exists in some leading mainstream products in the world. A main level of cross power is that bypass method may be used when intersection is carried out and main wave length intersection is an important expression of cross level. Main capacity of intersection is 6.4 Tbit per second-12.8Tbit per second. It is an exchange optical technique method in the optical layer.

4. Being applied to integration between PTN network and transport network

Simultaneous development of transport network and PTN network is a quite advanced strategic method at present. PTN grouping transmission plays an important role in gradual integration towards an optimized direction and reduction in overall cost of operation network and capital expenditure. Primarily, the important network that carries out transmission on electric/ optical control plane improves intelligent and dynamic mobilization of network service effectively and can provide some new services or recover service. Later, it transfers to a larger development direction and bigger particles. In such a process, direction upgrade transition transport layer for PTN can be finished gradually and PTN transmission nodes can be used to lead and support metropolitan area convergence network. Ability transmission is also contained. Thus, it can break and make up limitations and shortages of transmission network of the previous two-layer data network effectively as well as construct uniform network convergence performance efficiently.

\section{Conclusion:}

In the $21^{\text {st }}$ century, electronic information technology has obtained significant performance in the whole world and driven development of mobile communication network largely. With development of the era, people propose higher requirements for communication quality and rate. Under this background, a kind of rapider and more convenient $4 \mathrm{G}$ mobile communication network appears in our life and work. Besides, optical communication technique has been widely applied to 4G mobile communication network. Development of communication network and that of optical communication promote and adapt to each other. In both foreign countries and China, optical 
communication equipment plants and operators also work hard to drive continuous development and progress in the technique. Application of optical communication technique to $4 \mathrm{G}$ network improves speed of information dissemination continuously, re-expands capacity of communication techniques constantly, strengthens information service quality of globality, shows advantages of $4 \mathrm{G}$ techniques in many aspects and drives development of current communication technology.

\section{References}

[1] Geng Jun, Ma Donghui. Analysis of application of optical communication technique to 4G development [J]. World of Electronics and Network, 2013(08).

[2] Liu Jia. Discussion on PTN and OTN in local metropolitan area transmission networking models [J]. Telecommunication Bulletin, 2011(02).

[3] An Zhiqiang. Research on optical communication technique combining orthogonal frequency division multiplexing in electrical field with fibre optical communication technology [J]. Guide of Sci-tech Magazine, 2014(06).

[4] Xie Wenhui. Primary exploration on application and development of $4 \mathrm{G}$ technique in modern communication $[\mathrm{J}]$. Analysis about Development and Application of Contemporary Information and Communication Technology, 2011(06).

[5] Yang Xuezhou, Tang Wei, Guo Wei. Research on OFDM cognitive network resource allocation algorithm based on practical application environment [J]. Application Research of Computers, 2010(02).

[6] Rong Jian, Han Jiajia, Zhong Xiaochun. Development and key techniques of deep space exploration optical communication technique $[\mathrm{J}]$. Conference proceedings of the $2^{\text {nd }}$ academic conference held by Committee of Deep Space Exploration Technology, Chinese Society of Astronautics, 2010(01).

[7] Ning Jiajun. Research on promoting development of 4G network technology under optical communication technique $[\mathrm{J}]$. Development and Application of Communication Technology, 2011(05).

[8] Zhang Zhen. A new trend of optical communication technique development [J]. Analysis of Historical Background, 2011(08).

[9] Liu Jianming, Zhao Feng, Zhang Yuexia, Cui Qimei, Hu Pingping. Application of 3G and 4G wireless communication technique to ICT network mode [J]. Power System Communication, 2009(07).

[10] Zhou You, Zhu Shilei, Hu Han Ying. A new frequency offset estimation algorithm of constant modulus signal OFDM system [J]. Journal of Jilin University (Engineering Version). 